\title{
Maxillofacial surgery and COVID-19, The Pandemic !!
}

\author{
Rishi Kumar Bali ${ }^{1} \cdot$ Kirti Chaudhry $^{2}$
}

Published online: 11 April 2020

(c) The Association of Oral and Maxillofacial Surgeons of India 2020

The pandemic of novel coronavirus disease (COVID-19) is having an unprecedented impact on all aspects of our lives. Never in the history of mankind, have we faced a global public health issue of this enormous magnitude with its impact across a spectrum of economic activity, travel, governance, education, and the like.

As the world responds to this crisis with behavior modifications like social distancing, lockdowns, and quarantine of suspected cases, healthcare providers are at the frontline in the fight against the coronavirus. The pandemic has deeply affected the delivery of services by the various healthcare specialities. We maxillofacial surgeons are particularly vulnerable to transmissible diseases by way of droplet transmission both due to the area of work and the type of instrumentation.

The long and unpredictable incubation periods (0-27 days, mean 6.4 days) [1] of the virus in COVID-19 patients expose us to the risk of treating asymptomatic patients. Worse still, many patients who are harboring the virus may conceal their flu like symptoms or might be convalescing from the disease.

Rishi Kumar Bali

rshbali@yahoo.co.in

Kirti Chaudhry

Chaudhry_kirti@yahoo.com

1 DAV Dental College and MDM General Hospital, Yamunanagar, India

2 Department of Dentistry, All India Institute of Medical Sciences, Jodhpur, India

\section{So How Do We Go About Fulfilling Our Professional Obligations?}

As professionals, we are always rigorous about infection control. Nevertheless, it is worthwhile to review our current practices of infection control to tighten them even further. The COVID-19 can remain infectious on inanimate surfaces for $2 \mathrm{~h}$ up to 9 days, depending on the temperature, humidity, type of surface and viral load [2].

It takes the entire team to avert cross-infection-staff, nurses, paramedics, trainees, and waste handlers. The head of the maxillofacial surgery unit should use the opportunity to reiterate and reinforce the best practices in hospital infection control including the use of hand hygiene practices, barrier techniques, the use of personal protective equipment (PPE), disinfection of surfaces, and sterilization of equipment. Appropriate use of disinfectants and methods of mopping of surfaces like the triple-bucket method should be monitored at the highest level. Sodium hypochlorite $0.1 \%$ for $1 \mathrm{~min}$ has found to be effective against coronavirus [2]. The collection, segregation, and transport of infectious waste from COVID-19 patients should be done under the guidance of the Infection Control/ Biomedical Waste Management Officer of the institute.

Telescreening, telemedicine, and triaging will have to be put to optimal use in the Covid scenario. The Medical Council of India (MCI) has recently framed guidelines for the practice of telemedicine allowing telephonic and online consultations [3]. These shall decrease the footfall in the departments and enable the surgeon to decide when an operative intervention is necessary. Most maxillofacial surgeons are well trained with the operational triage protocols to be adopted in scenarios of mass disasters, wars, or accidents. 
Patients should be encouraged to seek online and telephonic consultations by displaying telephone numbers on departmental websites. While consulting, it is important to ask relevant history including travel history to COVID-19affected locations, the presence of symptoms such as fever, shortness of breath, cough, and loss of smell/taste.

Positive responses to either of the questions should raise concern, and elective care should be postponed for 3 weeks.

We should exert our professional discretion and avoid elective procedures at all costs to protect our community, our patients, our staff and ourselves. Exceptions are only to be made for emergency care. Both the CDC (Centers for Disease Control) [4] and the AOCMF [5] have recommended that all elective procedures should be postponed/ rescheduled during the pandemic till clear management strategies are identified.

Operative interventions should be limited to management of maxillofacial trauma including emergent airway management and bleeding, patients who need drainage of infections (e.g., Ludwigs angina) and oncosurgery procedures where a delay in management would adversely affect the outcome or could cause a permanent disability will need urgent attention.

The reverse transcriptase PCR using throat/nasal swabs or serological tests for COVID-19 test may not be available at most centers; when available, they may take 24-48 h. Since prompt intervention may be required in cases of severe infections or pan-facial trauma (particularly patients with bleeding, altered sensorium, or unconscious patients), it would be safer to assume them to be Covid positive till proven otherwise. It also needs to be cautioned that negative results do not preclude COVID-19 infection and should not be used as the sole basis for patient management decisions. Negative results should be combined with clinical observations, radiologic findings (CT scans of the lungs to look for any active lung involvement), patient history, and epidemiologic information.

As with most respiratory infections, droplets are the main source of transmission from COVID-19 patients. While treating COVID-19 positive or suspected patients, the N 95 (FFP2) [5] masks plus face shield should be used as a minimum requirement, during aerosol and surgical smoke generating procedures (like the use of handpieces, electrocautery, and ultrasonic instruments). It is important to mention that N95 masks were not found to be adequate to prevent transmission in Chinese surgeons and PAPR's (powered air purifying respirators) had to be used to prevent transmission from Covid patients [5]. Further, triple layered protection gowns and head caps are recommended to prevent fomite-based transmission.

Studies have shown that SARS and MERS were highly susceptible to Betadine rinse [6] and it can be assumed that a preprocedural rinse with the agent might reduce the load of coronaviruses in saliva. Povidone Iodine has been shown to have significant virucidal activity for about three hours and recently it has been recommended to coat the oral cavity and nasal passages of both the patient and the operating team before the procedure [7].

Intraoral radiographs like IOPA or occlusal views can stimulate gag reflexes and induce coughing should be avoided and substituted by extraoral techniques like OPG and cone beam CT.

On the clinical side, conservative treatment to preserve form and function must be instituted. Closed reduction of fractures (using IMF screws, Bridle wire stabilization or Eyelet wiring) should be preferred over open surgery where stability can be achieved without ORIF. This shall shorten operating time and facilitate early discharge. In situations where ORIF is absolutely necessary, transcuataneous approach (after applying a bio-occlusive dressing over the mouth post IMF) should be preferred over an intraoral approach [5].

Whenever possible patients with suspected or confirmed COVID-19 infection should not be treated in a routine practice setting, instead, they should only be managed in negative pressure theaters or airborne infection isolation rooms AIIRs.

Intubation should be performed by an experience anesthetist to limit the number of attempts and also in a manner that generates less coughing. The surgical team should enter the theater after 20 min of intubation with appropriate PPE to minimize the aerosol-based transmission. When doing open surgery, scalpel should be preferred over monopolar cautery and repeated suction/irrigation should be minimized; and when achieving hemostasis with bipolar cautery lowest power settings should be used. Absorbable sutures should be preferred to minimize unnecessary trips for their removal.

In sum, the COVID-19 situation continues to evolve, and given the significant ongoing research throughout the world, we as Maxillofacial surgeons have to remain updated to use the information to help our patients in a way that we minimize risk to the operating team and also help prevent community spread.

\section{Compliance with Ethical Standards}

Conflict of interest The authors declare that there is no conflict of interest.

\section{References}

1. https://www.worldometers.info/coronavirus/coronavirus-incuba tion-period. Accessed 30 Mar 2020 
2. Kampf G, Dodt T, Pfaeder S, Steinmann E (2020) Persistence of corona viruses on animate surfaces and their inactivation with biocide agents. J Hosp Infect 104:246

3. https://www.mohfw.gov.in/pdf/Telemedicine.pdf. Accessed 30 Mar 2020

4. https://www.cdc.gov/oralhealth/infectioncontrol/statementCOVID.html. Accessed 30 Mar 2020

5. https://aocmf3.aofoundation.org/\#o=News\%20Date\%20Facet,Des cending. Accessed 30 Mar 2020

6. Eggers M, Koburger-Janssen T, Eickmann M, Zorn J (2018) In vitro bactericidal and virucidal efficacy of povidone-iodine gargle/mouthwash against respiratory and oral tract pathogens. Infect Dis Ther 7:249-259

7. Kirk-Bayley J, Challacombe S, Sunkaraneni V, Combes J (2020) The use of povidone iodine nasal spray and mouthwash during the current COVID-19 pandemic may protect healthcare workers and reduce cross infection. https://doi.org/10.2139/ssrn.3563092

Publisher's Note Springer Nature remains neutral with regard to jurisdictional claims in published maps and institutional affiliations. 